Wiederaufforstung der gerodeten Fläche dieser qualitativ und quantitativ im Wesentlichen entspricht, erfolgt nicht. Die bauplanungsrechtliche Ausweisung reduziert die Anforderungen einer Wiederherstellungspflicht, wie dargelegt, nicht.

Bereits aufgrund des Baumalters wird die Zulassung des vorzeitigen Beginns den hier festgestellten Anforderungen an die Wiederherstellungspflicht nicht gerecht. In den Beschlüssen wird die Wiederherstellbarkeit der Fläche nicht hinreichend gewürdigt und auf die abschließende Erteilung der artenschutzrechtlichen Ausnahmegenehmigung wird nicht eingegangen. Insofern bestehen Zweifel an der Richtigkeit der Entscheidungen.

Allerdings erscheint nach überschlägiger Prüfung vorliegend für den Fall einer Genehmigungsversagung eine nachträgliche Legalisierung des geschaffenen Zustands durch Erteilung einer gesonderten Waldumwandlungsund Eingriffsgenehmigung einschließlich artenschutzrechtlicher Ausnahme für möglich. Aufgrund der bauplanungsrechtlichen Ausweisung ist der Eingriff auch ohne das immissionsschutzrechtliche Vorhaben vermutlich als nicht vermeidbar zu bewerten.

\section{Zusammenfassung}

Zusammenfassend lässt sich festhalten, dass die Anwendbarkeit von $\$ 8$ a BImSchG auf Rodungsmaßnahmen einzelfallabhängig ist. Es wurde gezeigt, dass eine naturschutzrechtliche Kompensation für die Erfüllung der Wiederherstellungspflicht aus $₫ 8$ a Abs. 1 Nr. 3 BImSchG unzureichend ist. Es bedarf der Herstellung eines dem früheren Zustand qualitativ und quantitativ im Wesentlichen entsprechenden Zustands.

Bei Waldflächen kann eine Wiederaufforstung zur Erfüllung der Wiederherstellungspflicht ausreichend sein, wenn die Wiederaufforstung der früheren Waldfläche vom durchschnittlichen Bestandsalter, der Altersstruktur, Besatzdichte und Artenzusammensetzung der Bäume sowie der Ausprägung der Strauchschicht im Wesentlichen entspricht; die Anpflanzung wesentlich jüngerer Bäume hingegen genügt nicht. Gegebenenfalls treten im Einzelfall weitere Aspekte hinzu. Eine Wiederherstellung muss hierbei mit vertretbarem Aufwand und verhältnismäßig kurzfristig - nicht erst nach mehreren Jahren - realisierbar sein. Sollte dies technisch nicht möglich oder wirtschaftlich nicht vertretbar sein, kommt eine Zulassung des vorzeitigen Beginns nicht in Betracht. In Bezug auf Rodungsmaßnahmen dürfte die praktische Bedeutung der Zulassung des vorzeitigen Beginns damit im Wesentlichen auf junge Waldflächen, Schlagfluren, Windwurf- oder ähnliche Flächen beschränkt sein.

Die im Verfahren Tesla für den Fall der Genehmigungsversagung vorgesehene Wiederaufforstung entspricht der gerodeten Waldfläche nicht im Wesentlichen und erfüllt damit nicht die in diesem Beitrag festgestellten Anforderungen an die Wiederherstellungspflicht. An der Wiederherstellbarkeit bestehen insbesondere aufgrund des Bestandsalters der gefällten Bäume Zweifel. Zudem umfasst die erteilte Zulassung nicht nur vorläufige Regelungen, sondern auch eine abschließende bzw. endgültige artenschutzrechtliche Ausnahme und überschreitet damit die Möglichkeiten des $\$ 8$ a BImSchG.

Die Beschlüsse des VG Frankfurt (Oder) und des OVG Berlin-Brandenburg setzen sich mit der Wiederherstellbarkeit der Waldfläche nicht hinreichend auseinander. Zwar stellt das OVG zutreffend fest, dass eine Anwendung des $\$ 8 \mathrm{a}$ BImSchG bei Waldflächen nicht grundsätzlich ausscheidet und dass in Anbetracht der Wiederaufforstungsmöglichkeit auf die Beschaffenheit der Waldfläche abzustellen ist. Eine konkrete Auseinandersetzung mit der Wiederherstellbarkeit der Waldfläche im vorliegenden Fall und eine Darlegung, ob die vorgesehene Wiederaufforstung dem früheren Wald qualitativ und quantitativ im Wesentlichen entspricht, unterbleibt allerdings. Auf die abschließende, verbindliche Erteilung der artenschutzrechtlichen Ausnahme im Rahmen der nur vorläufigen Zulassung des vorzeitigen Beginns gehen die Beschlüsse ebenfalls nicht ein. Insofern bestehen im Ergebnis Zweifel an deren Richtigkeit. Für den Fall der Genehmigungsversagung erscheint eine nachträgliche Legalisierung des geschaffenen Zustands jedoch nach überschlägiger Prüfung möglich.

\title{
Düngung bleibt weiterhin eine ökologische, rechtliche und politische Herausforderung*
}

\section{Stefan Möckel und André Wolf}

(c) Der/die Autor(en) 2020. Dieser Artikel ist eine Open-Access-Publikation.

In der öffentlichen Debatte ist die landwirtschaftliche Düngung bisher vor allem ein Problem der Nitrat-Richtlinie sowie der Gewässer und Grundwasserkörper. Dies verkürzt sowohl die vielfältigen ökologischen Wirkungen als auch die rechtliche Relevanz von Düngung. In Folge dieser eingeschränkten Betrachtung bleiben auch die neuesten Verschärfungen der Düngeverordnung hinter dem ökologisch Notwendigen sowie dem völker-, europa- und verfassungsrechtlich Gebotenen zurück. Ökologisch wie rechtlich sind zwei Hauptaufgaben zu lösen: die Reduzierung der anthropogenen Gesamtnährstoffemissionen und ein ausreichender Schutz lokaler

Dr. iur. Stefan Möckel und

Ass. jur., B. Sc. Geogr. André Wolf,

Helmholtz-Zentrum für Umweltforschung - UFZ,

Leipzig Deutschland
Ökosysteme vor übermäßigen Nährstoffeinträgen entsprechend ihrem Erhaltungszustand und ihrer spezifischen Vulnerabilität. Diese Aufgaben erfordern einen breit aufgestellten Instrumentenmix, der weit über das gegenwärtige Düngerecht hinausgeht.

\section{Nährstoffüberschüsse als ökologische, politische und rechtliche Herausforderung}

Das Bild Deutschlands als Industrieland ist geprägt von Fahrzeug- und Anlagenbauern sowie Herstellern chemi-

\footnotetext{
^ Diese Arbeit wurde auch mitfinanziert vom Bundesministerium für Bildung und Forschung (BMBF) im Rahmen der Förderinitiative „Boden als nachhaltige Ressource für die Bioökonomie BonaRes“, Förderkennzeichen 031B0511A.
} 
scher Erzeugnisse. Die landwirtschaftliche Nutzung auf mehr als 50 Prozent der Landfläche ${ }^{1}$ wird hierbei meist übersehen, obwohl auch hier überwiegend eine industriell geprägte Bewirtschaftung stattfindet. Technischer Fortschritt bei Dünge- und Pflanzenschutzmitteln, landwirtschaftlichen Maschinen, Anbaumethoden sowie Züchtungsfortschritte und die ertragsorientierte Umgestaltung der Agrarlandschaften haben in den letzten 100 Jahren die Erträge, aber auch die negativen Umweltauswirkungen um ein Vielfaches ansteigen lassen. ${ }^{2}$

Hierbei kommt dem Wandel von einer noch nährstofflimitierten Landwirtschaft am Beginn des 20. Jahrhundert hin zu einer Nährstoffüberschusswirtschaft eine entscheidende Bedeutung zu. ${ }^{3}$ Diese Entwicklung wurde zum einen durch die Herstellung von mineralischen Stickstoffdünger mit Hilfe der Ammoniaksynthese im Haber-Bosch-Verfahren ermöglicht. ${ }^{4} \mathrm{Zum}$ anderen durch die Ausweitung des Welthandels, sowohl von Düngemitteln (z. B. Guano, Phosphor) als auch Futtermitteln (insbesondere Soja aus Süd- und Nordamerika). Künstlich hergestellte und importierte Düngemittel sowie zugekaufte Futtermittel bewirken eine Durchbrechung der regionalen Nährstoffkreisläufe in der Landwirtschaft und sind die Hauptursache für die hohen Stickstoffüberschüsse von durchschnittlich rund $100 \mathrm{~kg}$ pro Hektar und Jahr (siehe Abbildung 1). ${ }^{5}$ Insgesamt verbleibt rund die Hälfte des zugeführten Stickstoffs als Überschuss in Böden, Gewässern, Meeren und als klimarelevantes Lachgas in der Atmosphäre. Für ganz Deutschland bedeutet dies ein Zuviel an reaktivem Stickstoff in Höhe von jährlich über 1.5 Mio. kg. ${ }^{6}$ Allerdings bestehen hierbei größere regionale Unterschiede. ${ }^{7}$ Auch die Art der Bewirtschaftung ist entscheidend. Gegenüber dem konventionellen Landbau weist eine Bewirtschaftung nach den Kriterien des ökologischen Landbaus im Regelfall sowohl geringere Stickstoffüberschüsse je Hektar als auch eine höhere Stoffeffizienz je Ertrag auf. ${ }^{8}$

Der Einsatz von mineralischem Stickstoffdünger ${ }^{9}$ hat zugleich einen erheblichen Anteil daran, dass sich die Landwirtschaft in Deutschland im 20. Jahrhundert energetisch von einer Primärproduktion zu einer Bewirtschaftung mit großem Input an fossilen Energien wandelte. Schon 1985 konstatierte Hermann Priebe: ,Jedes Jahr flieBen 16 Milliarden Mark in unsere Landwirtschaft. Das ist weit mehr, als sie erwirtschaftet. Noch grotesker: In Kalorien gerechnet, verbrauchen Landwirte mehr Energie als sie erzeugen." 10 Ursächlich ist v. a. das Haber-Bosch-Verfahren, welches rund 3-5\% der weltweiten Erdgasförderung bzw. 1-2\% des weltweiten Energiebedarfs benötigt und für eine Tonne Ammoniakertrag rund 1,5 Tonnen $\mathrm{CO}_{2}$ freisetzt. ${ }^{11}$ Weltweit werden seit 2013 jährlich über 140 Mio.t Ammoniak für Düngemittelproduktion hergestellt und damit mehr als 200 Mio. t $\mathrm{CO}_{2}$ emittiert. $^{12}$ Gleichwohl spielt die Reduzierung des Einsatzes von mineralischen Stickstoffdünger in dpolitischen Debatte um eine klimaschonende Landwirtschaft bisher keine Rolle. ${ }^{13}$ Dabei zeigt der ökologische Landbau, bei dem mineralischer Stickstoffdünger nicht gestattet ist, ${ }^{14}$ dass eine Landwirtschaft mit niedrigerem Energieeinsatz je Hektar und einer höheren Energieeffizienz je Ertrag durchaus möglich ist. ${ }^{15}$ Insbesondere der Einsatz von stickstoffbindenden Leguminosen als Zwischenfrüchte spart dabei nicht nur Energie und $\mathrm{CO}_{2}$-Emissionen ein, ${ }^{16}$ sondern erhöht zugleich auch die Humus- und Kohlenstoffgehalte in Böden mit positiven Wirkungen für Wasserrückhalt, Bodenfruchtbarkeit und das Klima. ${ }^{17}$

Insgesamt sind Einträge an reaktivem Stickstoff und Phosphor in Deutschland im globalen und europäischen Vergleich sehr hoch und überschreiten die planetaren Belastungsgrenzen der Ökosysteme. ${ }^{18}$ Diese Situation hat sich bzgl. Nitrat bis 2018 auch nicht signifikant verbessert. Der Anteil grenzwertüberschreitend gemessener Grundwässer verharrt mit $26,7 \%$ auf annähernd gleichbleibendem $\mathrm{Ni}$ - veau; die Küsten- und Meeresgewässer halten die Orientierungs- und Schwellenwerte gemäß WRRL resp. MSRL ganz überwiegend deutlich nicht ein und müssen als weiträumig eutrophiert eingestuft werden. ${ }^{19}$ Nur bei den Oberflächenfließ- und Stillgewässern gibt es deutlichere Verbes-

1) Röder u. a., Status quo und aktuelle Entwicklungen der landwirtschaftlichen Flächennutzung in Deutschland, Natur und Landschaft 2018, $250 \mathrm{ff}$.

2) BMEL, Landwirtschaft verstehen - Fakten und Hintergründe, 2018; Heißenhuber/Haber/Krämer, 30 Jahre SRU Sondergutachten „Umweltprobleme der Landwirtschaft” - eine Bilanz, 2015; Uekötter, Die Wahrheit ist auf dem Feld. Eine Wissensgeschichte der deutschen Landwirtschaft, 2010.

3) Uekötter, 2010 (Fn. 2).

4) Ammoniak ist Voraussetzung für mineralischen Stickstoffdünger wie z. B. Harnstoff, Ammoniumnitrat. Vgl. UBA, Daten zur Umwelt: Umwelt und Landwirtschaft, 2018, S. 58.

5) Нäußermann u.a., Stickstoff-Flächenbilanzen für Deutschland mit Regionalgliederung Bundesländer und Kreise - Jahre 1995 bis 2017, 2019, S. 76 ff.; UBA, 2018 (Fn. 4), S. 19 ff., $57 \mathrm{ff}$. Vgl. auch BMEL/BMU 2020, Nitratbericht 2020, S. 82 f. mit der abweichenden Bilanzierung nach der Flächenbilanzmethode.

6) BMEL, Nährstoffbilanz insgesamt von 1990 bis 2017, https://www. bmel-statistik.de/fileadmin/daten/MBT-0111290-0000.xls 2019.

7) Häußermann u.a., 2019 (Fn. 5), S. 79 ff.; vgl. auch BMEL/BMU 2020 (Fn. 5), S. 42, Abb. 20.

8) Thünen-Institut, Leistungen des ökologischen Landbaus für Umwelt und Gesellschaft, Thünen Report 65, 2019; Mäder u.a., Soil Fertility and Biodiversity in Organic Farming, Science 2002, $1694 \mathrm{ff}$; darüber hinaus erweist sich der Ökolandbau als eine sehr kostenwirksame Maßnahme zur Minderung der Stickstoffsalden/des Stickstoffaustrags ( $€ / \mathrm{kg} \mathrm{N} / \mathrm{ha}$ ), vgl. BMEL/BMU 2020 (Fn. 5), S. 99-101 m.div.Nw.

9) Zur räumlichen Verteilung in Deutschland siehe Häußermann u. a., 2019 (Fn. 5), S. 86

10) Priebe, Die subventionierte Unvernunft - Landwirtschaft und Naturhaushalt, 1985, Cover und S. 56.

11) Smith/Hill/Torrente-Murciano, Current and future role of HaberBosch ammonia in a carbon-free energy landscape, Energy \& Environmental Science 2020, https://doi.org/10.1039/C9EE02873K Pfromm, Towards sustainable agriculture: Fossil-free ammonia, Journal of Renewable and Sustainable Energy 2017, https:// doi.org/10.1063/1.4985090; Smith, Nitrogenase Reveals Its Inner Secrets, Science 2002, https://doi.org/10.1126/science.1076659; Smil, Enriching the earth: Fritz Haber, Carl Bosch, and the transformation of world food production, 2004. Weltweit wurden seit 2013 jährlich über 140 Mio.t Ammoniak für Düngemittelproduktion hergestellt (International Fertilizer Association Production \& Trade Tables by Region, https://www.ifastat.org/supply/ Nitrogen\%20Products/Ammonia 2019).

12) International Fertilizer Association 2019 (Fn. 11).

13) So beschränkt sich z.B. das Klimaschutzprogramm der Bundesregierung auf eine effizientere Düngung und eine Reduzierung der Lachgasemissionen mittels besserer Ausbringungstechniken (Bundesregierung, Klimaschutzprogramm 2030 der Bundesregierung zur Umsetzung des Klimaschutzplans 2050, 2019, S. 102ff.).

14) In der EU gemäß Art. 12 EU-Verordnung 834/2007.

15) Thünen-Institut, 2019 (Fn. 8).

16) Badgley u.a., Organic agriculture and the global food supply, Renewable Agriculture and Food Systems 2007, https:// doi.org/10.1017/s1742170507001640; Smith Science 2002, 1654 f.

17) Kallenbach u. a., Microbial physiology and necromass regulate agricultural soil carbon accumulation, Soil Biology and Biochemistry 2015, https://doi.org/10.1016/j.soilbio.2015.09.005; McDaniel/ Tiemann/Grandy, Does agricultural crop diversity enhance soil microbial biomass and organic matter dynamics? A meta-analysis, Ecological Applications 2014, https://doi.org/10.1890/13-0616.1; Stockmann u.a., The knowns, known unknowns and unknowns of sequestration of soil organic carbon, Agriculture, Ecosystems \& Environment 2013, https://doi.org/10.1016/j.agee.2012.10.001.

18) Steffen u.a., Planetary boundaries: Guiding human development on a changing planet, Science 2015, 736ff.; EEA, Agricultural land use intensity, http://www.eea.europa.eu/data-and-maps/figures/agricultural-land-use-intensity-1, 2015.

19) BMEL/BMU 2020 (Fn. 5), für Grundwasser: S. 5, 10, für Meeres- u. Küstengewässer: 48 ff. (61), 63 ff. (76f.). 


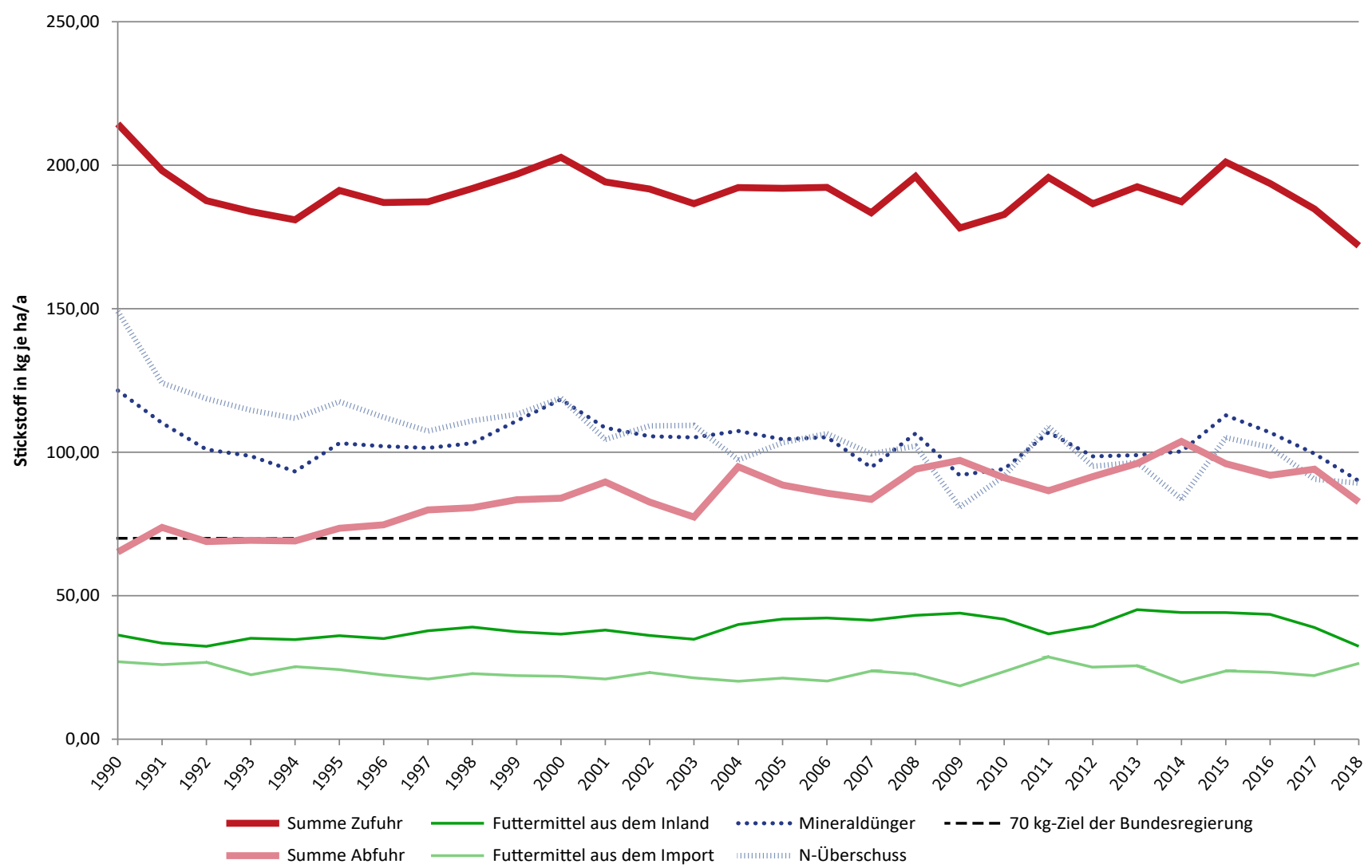

Abb. 1 Nährstoffbilanz für reaktiven Stickstoff in Deutschland in Kilogramm je Hektar und Jahr von 1990 bis 2018. Quelle: Eigene Darstellung nach Daten von BMEL und Bundesregierung..24

serungen für Nitrat und Phosphor mit lokal begrenzten Verschlechterungen. ${ }^{20}$

Trotz dieses hohen Intensivierungsgrads der deutschen Landwirtschaft leistet Deutschland entgegen mancher Behauptungen in der Summe keinen Beitrag zur Welternährung, da Deutschland - wie ganz Europa - mengenmäßig mehr Agrarprodukte importiert als es exportiert. ${ }^{21}$ Weniger die hohe Bevölkerungsdichte je $\mathrm{km}^{2}$ als die hohen Nutztierbestände und die hierfür benötigten Futtermittel sind Grund dafür, dass wir weltweit große Agrarflächen in anderen Staaten nutzen. ${ }^{22}$ Dies ermöglicht eine Überversorgung sowie erhebliche Exportüberschüsse bei tierischen Lebensmitteln, verursacht aber zugleich einen überhöhten Anfall an Wirtschaftsdünger, insbesondere in den norddeutschen und bayrischen Regionen mit hohen Nutztierbeständen und Biogasanlagen. ${ }^{23}$

\subsection{Düngung als politische Herausforderung}

Der Einsatz von Düngemittel wird in der Bundesrepublik Deutschland schon seit 1962 gesetzlich geregelt. ${ }^{25}$ Seit seinem ersten Umweltgutachten im Jahr 1974 weist der Sachverständigenrat für Umweltfragen regelmäßig auf die Umweltfolgen der sich intensivierenden Landwirtschaft und insbesondere auf die durch zu hohe Nährstoffeinträge entstehenden Eutrophierungsprobleme und Folgewirkungen hin. ${ }^{26}$ Dessen ungeachtet wurde 1976 die schon damals umstrittene Landwirtschaftsklausel ins neue Bundesnaturschutzgesetz (BNatSchG) aufgenommen, wonach die ,ordnungsgemäße land-, forst- und fischereiwirtschaftliche Bodennutzung nicht als Eingriff in Natur und Landschaft anzusehen" ist. ${ }^{27}$ Als freistellende Regelvermutung in $\int 14 \mathrm{Abs}$. $2 \mathrm{BNatSchG}$ hat sich diese seit Jahrzehnten empirisch ${ }^{28}$ und rechtlich ungerechtfertigte Privilegierung ${ }^{29}$ bis ins 21. Jahrhundert gehalten. Auch die Gemeinsame Agrarpolitik der EU ist in den letzten 50 Jahren mehrfach novelliert worden. Trotz Vorschriften zur Düngung und zur guten fachlichen Praxis im nationalen Recht steht eine deutliche Trendumkehr und dau-

20) BMEL/BMU 2020 (Fn. 5), für Nitrat: S. 28, 29 f., 36f., für Phosphor: S. $34 \mathrm{f} ., 39 \mathrm{f}$.

21) Vgl. UBA, 2018 (Fn. 4), S. 24f.; European Commission, Joint Research Centre, World Atlas of Desertification, Luxembourg 2018, S. 40 f.; Taube, Ökologische Intensivierung oder Erzeugung für den Weltmarkt?, 2019, https://www.acatech.de/wpcontent/uploads/2019/05/Taube_\%C3\%96kologische_Intensivierung_Weltmarkt.pdf.

22) UBA, 2018 (Fn. 4), S. $24 \mathrm{f}$

23) BMEL, Agrarexporte 2017 - Daten und Fakten, 2017; Häußermann u. a., 2019 (Fn. 5), S. $87 \mathrm{f}$.

24) BMEL, Nährstoffbilanz insgesamt von 1990 bis 2018, https:// www.bmel-statistik.de/fileadmin/daten/MBT-0111260-0000. xlsx, 2020; Bundesregierung, Deutsche Nachhaltigkeitsstrategie - Neuauflage 2016, S. 65.

25) Vgl. Douhaire, Rechtsfragen der Düngung: eine steuerungs- und rechtswissenschaftliche Analyse vor dem Hintergrund unionsund völkerrechtlicher Verpflichtungen und politischer Zielsetzungen zum Umwelt- und Ressourcenschutz, 2019, S. $92 \mathrm{f}$.

26) SRU/WBBGR, Für einen flächenwirksamen Insektenschutz, 2018; SRU, Stickstoff: Lösungsstrategien für ein drängendes Umweltproblem - Sondergutachten, 2015; SRU, Umweltgutachten 1994: Für eine dauerhaft-umweltgerechte Entwicklung, BT-Drs. 12/6995, 994; SRU, Umweltprobleme der Landwirtschaft - Sondergutachten, 1985; SRU, Umweltgutachten 1974 1974, Heißenhuber/Haber/Krämer, 2015 (Fn. 2).

27) SRU, Umweltgutachten 1978, 1978, Tz. 302.

28) Heinze u.a., Analyse der Gefährdungsursachen von Biotoptypen in Deutschland, Natur und Landschaft 2019; BfN, Daten zur Natur 2016, 2016; UBA, Umweltschutz in der Landwirtschaft, 2017.

29) Möckel, Gute fachliche Praxis, Eingriffsregelung und Landwirtschaft, NuR 2018, 742 ff.; Möckel, Landwirtschaft und naturschutzrechtliche Eingriffsgenehmigung - Anwendungsbereich und Verfassungsmäßigkeit der Regelvermutung sowie Erforderlichkeit pauschaler Kompensationspflichten, NuR 2012, $225 \mathrm{ff}$.; Brinktrine, Jahrbuch des Agrarrechts, 2003, $1 \mathrm{ff}$. 
erhafte Verbesserung der ökologischen Situation weiterhin aus. ${ }^{30}$

Düngung und Nährstoffüberschüsse sind in Deutschland spätestens seit Oktober 2016, als die Europäische Kommission vor dem Europäischen Gerichtshof (EuGH) ihre Klage wegen Nichtumsetzung der Richtlinie 91/676/EWG (Nitrat-Richtlinie) einreichte, zu einem Politikum geworden, wobei die Kommission schon 2011 eine Verschärfung des Düngerechts anmahnte. ${ }^{31}$ Trotz frühzeitiger Vorarbeiten $^{32}$ wurden erst 2017 das Düngegesetz (DüngG) ${ }^{33}$ und die Düngeverordnung $(\mathrm{DüV})^{34}$ novelliert sowie eine gesamtbetriebliche Brutto-Nährstoffbilanzierung eingeführt ( $(11 \mathrm{a}$ DüngG, Stoffstrombilanzverordnung - StoffBilV $\left.{ }^{35}\right)$. Dies hat die Verurteilung Deutschlands durch den EuGH wegen Verstoß gegen die europäische Nitrat-Richtlinie nicht mehr verhindern können. ${ }^{36}$ Auch wenn sich das Urteil auf das im Jahr 2016 geltende Düngemittelrecht bezieht, ist es zugleich für das aktuelle Düngerecht von Relevanz, da die Europäische Kommission nun prüfen muss, inwieweit die Novellierungen den Vertragsverstoß behoben haben oder ein zweites Verfahren vor dem EuGH zur Festsetzung von Vertragsstrafen nötig ist. Dass die Kommission die 2017er Novelle als nicht ausreichend einstufte, überraschte nicht, da etliche vom EuGH beanstandete Vorschriften und Regelungskonzepte beibehalten wurden ${ }^{37}$ und fraglich war, ob die neuen Vorschriften tatsächlich die Nitratbelastung von Oberflächengewässern und Grundwasserkörpern ausreichend verringern. ${ }^{38}$ Kurz vor Ausbruch der deutschen Corona-Krise hatte deshalb am 20.2.2020 das Bundeslandwirtschaftsministerium .. eine weitere Novellierung der DüV vorgelegt (siehe Überblick unter 2.), ${ }^{39}$ dem der Bundesrat am 27.3.2020 lediglich mit der Änderung zustimmte, dass die Verschärfungen für die roten Gebiete erst ab dem 1.1.2021 gelten sollen.

Die Europäische Kommission prüft nun erneut, ob Deutschland mit den Änderungen das EuGH Urteil und die Nitrat-Richtlinie nunmehr vollständig umsetzt. Aufgrund der Anerkennung eines Klagerechts von Umweltverbänden auch hinsichtlich der Nitrat-Richtlinie durch den EuGH ${ }^{40}$ dürfte die Kommission dabei kaum politische Kompromisse eingehen. Schon jetzt ist zu konstatieren, dass sowohl die Novelle als auch die politische Diskussion zu sehr auf die Nitrat-Richtlinie und den Schutz von Gewässern fokussiert war, weshalb die weitreichenden ökologischen Auswirkungen von Nährstoffüberschüssen für terrestrische Ökosysteme und das Klima (siehe 1.2) sowie diesbezügliche Schutzverpflichtungen Deutschlands (siehe 1.3) nicht in ausreichendem Maße angegangen wurden.

\subsection{Düngung als systemischer Umweltfaktor mit weitreichenden Auswirkungen}

Die landwirtschaftlichen Nährstoffüberschüsse, welche in den terrestrischen und aquatischen Ökosystemen verbleiben, verharren seit Jahren in Deutschland auf hohem Niveau (siehe Abbildung 1). ${ }^{41}$ Der hohe Eintrag an reaktivem Stickstoff in die Umwelt hat erhebliche negative Folgen für die betroffenen Gewässer, Grundwasserkörper, Biotope und wildlebenden Arten sowie für das globale Klima und die Nord- und Ostsee. ${ }^{42}$

In Böden und Gewässern bewirken hohe Stickstofffrachten eine Eutrophierung sowie Versauerung mit nachhaltigen Folgewirkungen für die biologische Vielfalt und die Mobilisierung von Schwermetallen. Schon jetzt sind in vielen Regionen Deutschlands die ökologischen Belastungsgrenzen für Eutrophierung und Versauerung überschritten und die Trinkwassergewinnung durch den Eintrag von Nitrat beeinträchtigt. ${ }^{43}$ Gasförmige Stickstoffemissionen befördern die Bildung von bodennahem Ozon sowie sekundären Feinstäuben und erhöhen zusammen mit Stickstoffdioxid die Belastungen für die menschliche Gesundheit. Sowohl Ozon als auch die Ammoniakemissionen aus Tier- haltung und Düngung, ${ }^{44}$ welche 95 Prozent der Gesamtemissionen ausmachen, schädigen Pflanzen unmittelbar.

Neben diesen direkten Folgen haben Nährstoffüberschüsse auch langfristige bzw. systemische Auswirkungen, insbesondere für die Atmosphäre und die Biosphäre. Die landwirtschaftlichen Emissionen von Lachgas $\left(\mathrm{N}_{2} \mathrm{O}\right)$ verstärken sowohl die Klimaerwärmung als auch den Abbau der Ozonschicht. Neuere Studie zeigen, dass die Emissionen weltweit deutlich ansteigen und Lachgas nicht nur die Klimabilanz von Bioenergie verhagelt, sondern mittlerweile sogar FCKW als Hauptursache für den Ozonabbau verdrängt hat. ${ }^{45}$ Lachgas entsteht in der Landwirtschaft v.a. durch die mikrobielle Umwandlung von reaktivem Stickstoff im Boden, wobei die Emissionen im ökologischen Landbau aufgrund der niedrigeren Stickstoffdüngung ca. $40 \%$ geringer sind als in der konventionellen Bodenbewirtschaftung. ${ }^{46}$

30) Heißenhuber/Haber/Krämer, 2015 (Fn. 2); umfassend zu Nitrat und bzgl. der Oberflächengewässer auch zu Phosphor BMEL/BMU 2020 (Fn. 5), wo deutlichere Verbesserungen und Trend nur bei den Oberflächengewässern berichtet werden.

31) $\mathrm{EuGH}, \mathrm{Klageschrift} \mathrm{der} \mathrm{Europäischen} \mathrm{Kommission} \mathrm{gegen} \mathrm{die} \mathrm{die}$ Bundesrepublik Deutschland vom 27.10.2016 - C-543/16.

32) WBA/WBD/SRU, Novellierung der Düngeverordnung: Nährstoffüberschüsse wirksam begrenzen, 2013; Bund-Länder-Arbeitsgruppe, Evaluierung der Düngeverordnung - Ergebnisse und Optionen zur Weiterentwicklung, 2012.

33) Düngegesetz vom 9.1.2009 (BGBl. I S. 54, 136) in der Fassung des Artikel 1 des Gesetzes vom 5. 5.2017 (BGBl. I S. 1068).

34) Verordnung über die Anwendung von Düngemitteln, Bodenhilfsstoffen, Kultursubstraten und Pflanzenhilfsmitteln nach den Grundsätzen der guten fachlichen Praxis beim Düngen vom 26.5.2017 (BGBl. I S. 1305).

35) BMEL, Verordnung über den Umgang mit Nährstoffen im Betrieb und betriebliche Stoffstrombilanzen vom 14. Dezember 2017 (BGBl. I S. 3942).

36) $\mathrm{EuGH}$, Urt. v. 21.6.2018 - C-543/16.

37) Ausführlicher UBA, Auswertung des Urteils des Europäischen Gerichtshofs (EuGH) vom 21.6.2018 in der RS C-543/16 wegen Vertragsverletzung (Nitratrichtlinie 91/676/EWG), 2018; Här$t e l$, Zwischen Grundwasserschutz und effizienter Landwirtschaft: zur Umsetzungsproblematik der Nitratrichtlinie, NuR 2019, 289 ff.; Douhaire, Schon wieder eine Novelle des Düngerechts? Das EuGH-Urteil vom 21.6.2018 zur Nitratrichtlinie und seine Folgen, ZUR 2018, $464 \mathrm{ff}$;; Möckel, Entspricht das neue deutsche Düngerecht den im EuGH-Urteil vom 21.6.2018 genannten Anforderungen?, NVwZ 2018, 1599 ff. Optimistischer Reinhardt, Schutz der Gewässer vor Nitrateinträgen aus landwirtschaftlicher Bodennutzung - Die neue Düngeverordnung nach dem Urteil des EuGH zur Umsetzung der Nitratrichtlinie, NuR 2019, $217 \mathrm{ff}$

38) Taube, Expertise zur Bewertung des neuen Düngerechts (DüG, DüV, StoffBilV) von 2017 in Deutschland im Hinblick auf den Gewässerschutz - Studie im Auftrag von: BDEW - Bundesverband der Energie- und Wasserwirtschaft e. V., 2018.

39) BR-Drs. 98/20, Verordnung zur Änderung der Düngeverordnung und anderer Vorschriften.

40) EuGH, Urt. v. 3.10. 2019 - C-197/18, NuR 2019, 751

41) UBA, 2018 (Fn. 4), S. 57 ff.

42) BMEL, Diskussionspapier: Ackerbaustrategie 2035 - Perspektiven für einen produktiven und vielfältigen Pflanzenbau, 2019; UBA, 2018 (Fn. 4), S. 57 ff.; Bundesregierung, Stickstoffeintrag in die Biosphäre, 2017; UBA, Reaktiver Stickstoff in Deutschland: Ursachen, Wirkungen, Maßnahmen, 2015; UBA, 2017 (Fn. 28); BfN, 2016 (Fn. 28).

43) UBA, 2018 (Fn. 4), S. $74 \mathrm{ff}$.

44) Нäußermann u. a., 2019 (Fn. 5), S. 89; UBA, 2018 (Fn. 4), S. $72 \mathrm{f}$.

45) Thompson u.a., Acceleration of global N2O emissions seen from two decades of atmospheric inversion, Nature Climate Change 2019; Ravishankara/Daniel/Portmann, Nitrous Oxide $\left(\mathrm{N}_{2} \mathrm{O}\right)$ : The Dominant Ozone-Depleting Substance Emitted in the 21st Century, Science 2009, https://doi.org/10.1126/science.1176985; Crutzen u. a., $\mathrm{N}_{2} \mathrm{O}$ release from agro-biofuel production negates global warming reduction by replacing fossil fuels, Atmos. Chem. Phys. 2008.

46) Skinner u.a., The impact of long-term organic farming on soilderived greenhouse gas emissions, Scientific Reports 2019. 
Stickstoffeinträge und -überschüsse gefährden weiterhin die Ökosystemintegrität, ${ }^{47}$ da sich die Artenzusammensetzung in Ökosystemen im Zuge einer größeren Verfügbarkeit an Nährstoffen verändert. Pflanzenarten, die gut mit Nährstoffmangel umgehen können, ${ }^{48}$ werden verdrängt durch Generalisten, die auf gute Nährstoffversorgung mit starkem Wachstum und Samenbildung reagieren. Mit den Floraveränderungen kommt es auch zu Verschiebungen bei den wildlebenden Tierarten. Sowohl im terrestrischen Bereich als auch in Gewässern verhindern daher zu hohe Nährstoffeinträge aus der Landwirtschaft ein Erreichen günstiger Erhaltungszustände. ${ }^{49} \mathrm{Im}$ Boden führt eine hohe Versorgung mit reaktiven Stickstoff in der Regel zu einer gesteigerten mikrobiologischen Aktivität aber auch zu einem Rückgang von Mykorrhiza-Pilzen, die in Symbiose mit den Pflanzen leben und vielfältige positive Effekte für die Gesundheit auch von Kulturpflanzen und Bäumen haben. ${ }^{50}$ Die Effekte variieren hierbei sowohl in Abhängigkeit von der ausgebrachten Menge als auch zwischen den unterschiedlichen mineralischen Stickstoffdüngern (z. B. Harnstoff, Ammoniumnitrat, -sulfat, -phosphat) sowie der $\mathrm{Zu}-$ sammensetzung von Wirtschaftsdünger (u.a. Nähr- und Schadstoffgehalte). ${ }^{51}$

Ebenfalls eher versteckter Natur ist der Effekt, dass eine hohe Stickstoffversorgung regelmäßig auch einen größeren Einsatz von Pestiziden erfordert. Ursächlich ist zum einen, dass durch die hohen Stickstoffgehalte verstärkt Beikräuter auflaufen und die Kulturpflanzen schneller wachsen, höhere Proteingehalte aufweisen und dadurch anfälliger für Pilzbefall, Schädlinge und Halmbruch werden. ${ }^{52} \mathrm{Zum}$ anderen können die negativen Auswirkungen von hohen Stickstoffgaben sowie Pestiziden auf die Bodenfauna und insbesondere auf Mykorrhiza-Pilze die Vitalität der Kulturpflanzen und ihre Resilienz gegenüber Krankheiten und Schädlingen beeinträchtigen, was u.a. den Pestizidbedarf zusätzlich erhöht. ${ }^{53}$ Düngung bestimmt daher auch den Umfang von Pflanzenschutzmaßnahmen.

Eine überwiegende Düngung mit mineralischem Stickstoffdünger oder der ebenfalls hoch konzentrierten, leicht löslichen Gülle bewirken bei einem langjährigen Einsatz, dass der Gehalt an organischer Substanz (Humus) im Boden und der darin organisch gebundene Stick- und Kohlenstoff stark abnimmt, da anders als bei Mist oder Leguminosen zum einen dem Boden weniger organische Reststoffe zugeführt werden und zum anderen der leicht verfügbare Stickstoff die mikrobielle Aktivität und damit die mikrobielle Kohlenstoffnutzung und N-Mineralisierung erhöht. ${ }^{54}$ Aufgrund des Verzichts auf mineralischen Stickstoffdünger und dem verstärkten Anbau von Leguminosen als Zwischenfrüchte ist bei Flächen des ökologischen Landbaus der Gehalt an organischem Kohlenstoff im Boden (SOC) regelmäßig höher als bei vergleichbaren konventionellen Flächen. ${ }^{55}$

Nährstoffeinträge wirken sich räumlich je nach den geologischen, topographischen, klimatischen und sonstigen ökologischen Standortverhältnissen sowie der Art der jeweiligen Ökosysteme höchst unterschiedlich aus. ${ }^{56}$ So sind die Niederschlagsverhältnisse entscheidend inwieweit die Einträge in Gewässer und Grundwasserkörper verdünnt werden, während die Bodenverhältnisse (z.B. Typ, Humusgehalt, Feuchte, Grundwasserhöhe) maßgeblich darüber bestimmen, wie schnell und stark Nährstoffe mineralisiert, ausgewaschen oder emittiert werden oder sich im Boden akkumulieren. Das Klima hat Einfluss auf das Wachstum und damit den Nährstoffbedarf der Kulturpflanzen aber auch auf die Mineralisationsgeschwindigkeit in Böden. Schließlich unterscheiden sich nicht nur die Ökosysteme, sondern auch die Erhaltungszustände und Vulnerabilitäten von Habitaten und wildlebenden Tierund Pflanzenarten in Abhängigkeit je nach Belastungssituation, Artenzusammensetzung und den spezifischen Le- bensraumansprüchen der Arten. In Deutschland gibt es 72 Leitbodentypen und noch mehr Bodentypen ${ }^{57}$ sowie 799 Einzellandschaften und 690 verschiedene Biotoptypen (ohne städtische Verdichtungsräume), von denen über 50 Prozent als naturschutzfachlich schutzwürdig einzustufen sind. ${ }^{58}$ Dreiviertel der Biotope in Deutschland stuft das Bundesamt für Naturschutz als gefährdet ein, wobei Dün-

47) UBA, Ökosystemintegrität, https://www.umweltbundesamt.de/ themen/luft/luftschadstoffe-oekosysteme/wirkungen-auf-oekosysteme/oekosystemintegritaet\#einleitung, 2020; Schröder u.a., Anwendung des Bewertungskonzepts für die Ökosystemintegrität unter Berücksichtigung des Klimawandels in Kombination mit Stoffeinträgen - Abschlussbericht, 2019.

48) Hierzu zählen viele Arten, die von der FFH-Richtlinie 92/43/ EWG in den Anhängen II und IV als besonders schutzbedürftig eingestuft sind.

49) BMU/BfN, Die Lage der Natur in Deutschland; BfN, 2016 (Fn. 28); Heinze u.a. NuL 2019, $453 \mathrm{ff}$.

50) Vgl. Van Bruggen u.a., One Health - Cycling of diverse microbial communities as a connecting force for soil, plant, animal, human and ecosystem health, Sci. Total Environ 2019, https:// doi.org/10.1016/j.scitotenv.2019.02.091; van der Linde u.a., Environment and host as large-scale controls of ectomycorrhizal fungi, Nature 2018; Creamer u. a., Ecological network analysis reveals the inter-connection between soil biodiversity and ecosystem function as affected by land use across Europe, Applied Soil Ecology 2016; Sobotik, Wurzeln - Grundlage unserer landwirtschaftlichen Produktion: Wurzeln haben viele Funktionen, LOP 2010, $35 \mathrm{ff}$.

51) Vgl. Ryan u.a., Rhizosphere engineering and management for sustainable agriculture, Plant and Soil 2009; de la Torre u. a., An approach for mapping the vulnerability of European Union soils to antibiotic contamination, SciTotEnv 2012.

52) Vgl. Finckh/Van Bruggen/Tamm, Plant diseases and their management in organic agriculture, 2015; Huber/Haneklaus, Managing nutrition to control plant disease, Landbauforschung Volkenrode 2007, 313; Hofmeester, Effects of fertilization on pests and diseses, Netherlands Journal of Plant Pathology 1992, 257 ff.; Culliney/Pimentel, Ecological effects of organic agricultural practices on insect populations, Agriculture, ecosystems \& environment 1986, $253 \mathrm{ff}$.

53) Vgl. Bergmann, Impacts of Pesticide Pollution on Soil Microbial Communities, Ecosystem Function and Human Health, Earth Systems and Environmental Sciences 2019; Brzozowski/Mazou$r e k$, A Sustainable Agricultural Future Relies on the Transition to Organic Agroecological Pest Management, Sustainability 2018, 2023; Altieri/Ponti/Nicholls, in: Gurr/Wratten/Snyder u.a., Biodiversity and insect pests: key issues for sustainable management, 2012, 72; de Vries u. a., Soil bacterial networks are less stable under drought than fungal networks, Nature Communications 2018; Lekberg/Koide, Is plant performance limited by abundance of arbuscular mycorrhizal fungi? A meta-analysis of studies published between 1988 and 2003, New Phytol 2005.

54) Vgl. Chen u.a., Soil biodiversity and biogeochemical function in managed ecosystems, Soil Research 2019; Kallenbach u. a. Soil Biology and Biochemistry 2015, 279ff.; Mulvaney/Khan/Ellsworth, Synthetic Nitrogen Fertilizers Deplete Soil Nitrogen: A Global Dilemma for Sustainable Cereal Production, Journal of Environmental Quality 2009; Khan u. a., The Myth of Nitrogen Fertilization for Soil Carbon Sequestration, Journal of Environmental Quality 2007.

55) Vgl. Thünen-Institut (Fn. 8); Kallenbach u.a. Soil Biology and Biochemistry 2015, 279 ff.; Stockmann u. a. Agriculture, Ecosystems \& Environment 2013, 80 ff.; Hülsbergen/Schmid, in: ThünenInstitut, Klimawirkungen und Nachhaltigkeit ökologischer und konventioneller Pilotbetriebe in Deutschland, 2013, 4-10.

56) Vgl. Bowles u.a., Addressing agricultural nitrogen losses in a changing climate, Nature Sustainability 2018; UBA, 2018 (Fn. 4), S. $74 \mathrm{ff}$.

57) BGR, Bodenübersichtskarte der Bundesrepublik Deutschland 1:1000000, 1995.

58) BfN, 2016 (Fn. 28), S. 30 ff. und 42 ff., wobei 89 Landschaften als „,besonders schutzwürdig“ (etwa 12,3 Prozent der Bundesfläche), 99 Landschaften als ,schutzwürdig“ (10,8 Prozent der Bundesfläche) und 273 Landschaften als ,,schutzwürdig mit Defiziten“ (31,6 Prozent der Bundesfläche) eingestuft werden. 
gung und zu hohe Nährstoffeinträge einen maßgeblichen Anteil hieran haben. ${ }^{59}$

In Anbetracht der systemischen und langfristigen Natur vieler ökologischen Auswirkungen von übermäßiger Düngung, ist eine wesentlich größere Reduktion der Nährstoffüberschüsse als bisher notwendig. Dies gilt sowohl hinsichtlich der Gesamtmengen als auch hinsichtlich der spezifischen Einträge in lokale Ökosysteme.

\subsection{Düngung als völker-, europa- und verfassungsrechtliches Rechtsproblem}

Nicht nur die ökologische Notwendigkeiten, sondern auch völker-, europa- und verfassungsrechtliche Verpflichtungen erfordern von Deutschland eine Reduzierung der hiesigen Nährstoffüberschüsse und zwar sowohl in ihrer Gesamtheit als auch für spezifische, lokale Ökosysteme. ${ }^{60}$ International hat sich Deutschland in verschiedenen $\mathrm{Ab}-$ kommen und Konventionen zum Schutz der Nord- und Ostsee $^{61}$, zum Schutz des Klimas ${ }^{62}$ und zum Schutz von Ökosystemen und wildlebenden Tier- und Pflanzenarten ${ }^{63}$ verpflichtet, die direkt oder mittelbar eine Reduzierung der Nährstoffeinträge und -überschüsse erfordern.

Ergänzend und konkretisierend hat die Europäische Union mit Zustimmung Deutschlands in Verordnungen und Richtlinien ebenfalls umfangreiche rechtlich verbindliche Ziele und Vorgaben mit Relevanz für die Düngung normiert und zwar nicht nur in Bezug auf Nitrat in Gewässern und Grundwasserkörpern. So sind u. a. die nationalen Gesamtemissionen an Stickoxiden und Ammoniak gemäß der NEC-Richtlinie 2016/2284/EU bis 2030 deutlich zu reduzieren sowie die Gehalte an Nitrat im Grundwasser und Oberflächengewässern gemäß der Nitrat-Richtlinie und der Grundwasser-Richtlinie 2006/118/EG auf maximal $50 \mathrm{mg} \mathrm{N} / 1 \mathrm{zu}$ begrenzen. Bei Oberflächengewässern und Meeresgewässern ist nach der Wasserrahmen-Richtlinie 2000/60/EG und der Meeresstrategie-Rahmenrichtlinie 2008/56/EG ein guter ökologischer Zustand zu erreichen sowie bei allen in Anhang 2 und 4 der FloraFauna-Habitat-Richtlinie 92/4/EWG (FFH-Richtlinie) aufgelisteten Arten und bei allen europäischen Vogelarten nach der Vogelschutz-Richtlinie 2009/147/EG ein günstiger Erhaltungszustand zu gewährleisten bzw. wiederherzustellen. Des Weiteren plant die Europäische Kommission gemäß ihrer im Mai 2020 vorgelegten Farm to Fork Strategie politische und auch gesetzliche Maßnahmen im Rahmen des Green Deal, um bis 2030 die Nährstoffüberschüsse in die Umwelt mindestens zu halbieren und den Einsatz von Düngemmitteln um wenigstens 20 Prozent zu reduzieren. ${ }^{64}$ Damit will die Kommission sowohl die Biodiversitätsziele erreichen als auch bis 2050 die Treibhausgasemissionen auf netto Null reduzieren. ${ }^{65}$ Regelungen zur ökologisch nachhaltigen Verwendung von Düngemitteln und ihrer klimaneutralen Herstellung enthält allerdings auch die neue Verordnung 2019/1009 mit Vorschriften für die Bereitstellung von EU-Düngeprodukten auf dem Markt nicht.

Die gegen Deutschland laufenden Vertragsverletzungsverfahren der Europäischen Kommission sowie neuere Urteile des $\mathrm{EuGH}^{66}$ verdeutlichen, dass Deutschland bisher dem europäischen Umweltrecht nicht gerecht wird. ${ }^{67}$ Insbesondere hat der EuGH nun ausdrücklich klargestellt, dass entsprechend seiner bisherigen Rechtsprechung auch zugunsten der Landwirtschaft und ihrer Nährstoffeinträge sowohl bei der Nitrat-Richtlinie als auch bei der FFH-Richtlinie pauschale Freistellungen nur sehr eingeschränkt zulässig sind und die Mitgliedstaaten die unterschiedlichen ökologischen Standortverhältnisse bei der Regulierung und Zulassung landwirtschaftlicher Bodennutzungen angemessen berücksichtigen müssen. ${ }^{68} \mathrm{Nach}$ der Nitrat-Richtlinie sind in allen von Eutrophierung betroffenen Gewässern und Grundwasserkörpern die Einträge zu reduzieren. Bei Natura 2000 Gebieten ist gemäß Art. 6 Abs. 3 FFH-Richtlinie mittels Verträglichkeitsprüfungen für Düngemaßnahmen oder Weidehaltung sicherzustellen, dass dadurch die Gebiete mit ihren spezifischen Erhaltungszielen nicht erheblich beeinträchtigen werden und das Erreichen günstiger Erhaltungszustände erschwert oder ausgeschlossen wird. ${ }^{69}$ Dabei sind nach dem EuGH bei ungünstigen Erhaltungszuständen die Möglichkeiten zur Genehmigung von Tätigkeiten, welche die ökologische Lage der betreffenden Gebiete beeinträchtigen können, zwangsläufig begrenzt. ${ }^{70}$

Deutschland hat sich 1994 Art. 20a Grundgesetz (GG) als Leitlinie staatlichen und gesellschaftlichen Handelns ein umfassendes Umweltstaatsziel gegeben, welches von all seinen Organen zu verfolgen ist und auch entsprechende staatliche Schutzmaßnahmen rechtfertigt. Neben diesen rechtlichen Verpflichtungen bestehen mit den internationalen

59) BfN, 2016 (Fn. 28), S. 32 ff.; Heinze u. a. Natur und Landschaft 2019, 453 ff., wonach u. a. bei den Stickstoff-empfindlichen Biotoptypen ca. 80 Prozent gefährdet sind.

60) Vgl. Garske, Ordnungsrechtliche und ökonomische Instrumente der Phosphor-Governance: Unter Berücksichtigung der Wirkungen auf Böden, Gewässer, Biodiversität und Klima, 2020, S. 147 ff.; Douhaire, 2019 (Fn. 25), S. 48 ff.; Möckel in Möckel u. a., Rechtliche und andere Instrumente für vermehrten Umweltschutz in der Landwirtschaft, 2014, S. 215 ff.; Härtel, Düngung im Agrar- und Umweltrecht - EG-Recht, deutsches, niederländisches und flämisches Recht, 2002.

61) Gemäß den Übereinkommen zum Schutz der Meeresumwelt des Nordost-Atlantiks (OSPAR) und zum Schutz der Ostsee (HELCOM).

62) Rahmenübereinkommen der Vereinten Nationen über Klimaänderungen (UNFCCC) mit dem 2015er Übereinkommen von Paris.

63) Konvention über die Biologische Vielfalt 1992 (CBD); Bonner Übereinkommen zur Erhaltung der wandernden wild lebenden Tierarten 1979 (CMS); Berner Übereinkommen über die Erhaltung der europäischen wildlebenden Pflanzen und Tiere und ihrer natürlichen Lebensräume 1979; Ramsar Übereinkommen über Feuchtgebiete, insbesondere als Lebensraum für Wasserund Watvögel, von internationaler Bedeutung 1971.

64) Europäische Kommission, „Vom Hof auf den Tisch“ - eine Strategie für ein faires, gesundes und umweltfreundliches Lebensmittelsystem, Brüssel 2020.

65) Vgl. Europäische Kommission, Vorschlag für eine Verordnung zur Schaffung des Rahmens für die Verwirklichung der Klimaneutralität und zur Änderung der Verordnung (EU) 2018/1999 (Europäisches Klimagesetz), COM(2020) 80 final.

66) EuGH, Urt. v. 21.6.2018 - C-543/16 zur deutschen Nichtumsetzung der Nitrat-Richtlinie; Urteil C-293/17 und C-294/17 zum Schutz von Natura 2000 Gebieten vor Stickstoffeinträgen u.a. aus der Landwirtschaft und Urteil vom 3.10.2019 - C-197/18 zu Klagerechten von Umweltverbänden bei der Nitrat-Richtlinie. Den Umweltschutz stärken dürfte der EuGH auch im vom Bundesverwaltungsgericht vorgelegten Verfahren C-535/18.

67) Ausführlicher Möckel NVwZ 2018, 1599 ff. und Möckel, Natura 2000 Verträglichkeitsprüfung: Neue Entscheidungen des EuGH verdeutlichen die Defizite der deutschen Rechtslage und Rechtspraxis, NuR 2019, $152 \mathrm{ff}$.

68) EuGH, Urt. v. 21.6.2018 - C-543/16, Rdnr. 91-93; 111-114; Urteil C-293/17 und C-294/17, Rdnr. 93-101, 109-120; EuGH, Urt. v. 10.1.2006 - C-98/03, Rdnr. 41; Urt. v. 26.5.2011C-538/09, Rdnr. $41 \mathrm{ff}$.

69) Lediglich im Fall einer seit 1992 gänzlich unveränderten Bewirtschaftung ist nach dem EuGH keine FFH-Verträglichkeitsprüfung nötig, wobei schon Anpassungen aufgrund technischer und rechtlicher Änderungen diesen Bestandsschutz entfallen lassen (EuGH, Urt. v. 7.11.2018 - C-293/17 und C-294/17, Rdnr. 86 und 2. Entscheidungsgrund). Es gilt dann aber weiterhin das Verschlechterungsverbot des Art. 6 Abs. 2 FFH-Richtlinie (umgesetzt mit $₫ 33$ BNatSchG), welches ein ähnliches Schutzniveau verlangt (EuGH, Urt. v. 7.11.2018 - C-293/17 und C-294/17, Rdnr. 86; EuGH, Urt. v. 14.1.2016 - C-399/14, Rdnr. 52 m.w. N.).

70) EuGH, Urt. v. 7. 11.2018 - C-293/17 und C-294/17, Rdnr. 103 
Nachhaltigkeitszielen (SDGs) ${ }^{71}$ sowie nationalen Nachhaltigkeitsstrategien und -programmen ${ }^{72}$ umfangreiche politische Zielfestsetzungen, die ebenfalls direkt oder mittelbar die landwirtschaftliche Düngung betreffen.

\section{Wichtigste Neuerungen und Defizite der 2020er Novelle}

Gemäß $\ 3$ Abs. 3 DüngG dürfen nach Maßgabe der DüV Düngemittel ,nur so angewandt werden, dass durch die Anwendung die Gesundheit von Menschen und Tieren nicht geschädigt und der Naturhaushalt nicht gefährdet werden“. Nach $₫ 11$ Abs. 1 DüngG sind hierbei auftretende Nährstoffverluste in die Umwelt so weit wie möglich zu vermeiden. Bei den konkretisierenden Anforderungen der DüV zur Düngebedarfsermittlung und den erlaubten Nährstoffüberschüssen spielen der Erhaltungszustand und die Vulnerabilität der jeweiligen Ökosysteme allerdings keine Rolle mehr.

Mit der 2020er Novelle der DüV wurde die flächenbezogene Nährstoffbilanzierung mit ihren pauschalen Obergrenzen nach $\$ \int 8,9$ DüV a.F. aufgehoben. Dies bedeutet aber weder, dass Überschüsse nicht mehr zulässig sind noch, dass die Höhe zulässiger Überschüsse nun von den ökologischen Erfordernissen am jeweiligen Schlag oder Standort abhängen. Vielmehr gilt nunmehr allein $₫ 6$ Stoffstrombilanzverordnung (StoffBilV), welche keine flächenbezogene, sondern eine gesamtbetriebsbezogene Nährstoffbilanzierung ohne Berücksichtigung der ökologischen Standortverhältnisse vorschreibt und bis 2023 gemäß \$11a Abs. 2 DüngG und $\int 1 \mathrm{Abs}$. 2 StoffBilV nur für tierhaltende Betriebe ab 50 Großvieheinheiten oder 30 Hektar bzw. wirtschaftsdüngeraufnehmende Betriebe mit Biogasanlage gilt. Nach agrarwissenschaftlicher Einschätzung können je nach Betrieb sowie gewähltem Bilanzwertverfahren die Stickstoffüberschüsse sogar höher als die bisher nach $\$ 9$ DüV erlaubten $50 \mathrm{~kg}$ ha/a im Dreijahresdurchschnitt sein. ${ }^{73} \mathrm{Hinzu}$ kommt, dass anders als bei der aufgehobenen Flächenbilanzierung ( $\$ 14$ Abs. 1 Nr. 9 DüV 2017) die Überschreitung der Bilanzobergrenzen nach der StoffBilV keine Ordnungswidrigkeit ist, sondern die Behörden lediglich gemäß \$S 6 Abs. 5, 8 Abs. 1 StoffBilV eine bußgeldbewehrte Beratungspflicht anordnen können. Trotz dieser bekannten Defizite ${ }^{74}$ wurde 2020 die StoffBilV nicht novelliert.

Verschärft wurden die Anforderungen an die Düngung in den sogenannten ,,roten Gebieten“"gemäß $\$ 13 \mathrm{a} A$ bs. 1 DüV, in welchen europäische Vorgaben zum Schutz von Grundwasser bzw. Oberflächengewässern vor Nährstoffeinträgen akut gefährdet sind. Nach $\ 13$ a Abs. 2 DüV ist nun u. a. die jährliche betriebliche Gesamtsumme des Stickstoffdüngebedarfs um 20 Prozent zu kürzen und statt der betriebsbezogenen gilt eine schlagbezogene Obergrenze für Wirtschaftsdünger in Höhe von $170 \mathrm{~kg}$ Gesamtstickstoff pro Hektar. Auch wenn dadurch möglicherweise gewisse Ertragsrückgänge entstehen, ist diese Verschärfung in Anbetracht der Stickstoffüberschüsse von im Schnitt $50 \%$ der zugeführten Düngemengen nicht nur zumutbar, sondern sogar ein Zugeständnis zugunsten der Landwirtschaft, welche das Risiko einer anhaltenden Zielverfehlung birgt.

Weitere Verbesserungen resultieren aus der Verkürzung der Einarbeitungszeit in $\$ 6 \mathrm{DüV}$ von vier auf eine Stunde, wobei eine sofortige Einarbeitung noch mehr Ammoniakemissionen verhindern würde. Auch die Ausweitung der Gewässerrandstreifen in $\int 5 \mathrm{DüV}$ schützt Oberflächengewässer besser vor Nährstoffeinträgen. Allerdings gelten die Abstandsgebote gemäß $₫ 5$ Abs. 4 DüV weiterhin nicht bei Kleingewässern (z.B. Entwässerungsgräben), die nach $\ 2$ Abs. 2 WHG von den Ländern vom Anwendungsbereich des WHG freigestellt werden, obwohl diese regelmäßig in größere Oberflächengewässer entwässern. ${ }^{75}$ Auch für Flächen mit entwässernden, unterirdischen Drainagen gelten nach wie vor keine besonderen Schutzanforderungen. ${ }^{76}$
Weiterhin bleiben etliche Fragen offen: ${ }^{77}$ Warum gibt es für Mineraldünger in Anbetracht der weitreichenden ökologischen Auswirkungen keine Ausbringungsobergrenzen? Warum sind bei der Bestimmung des Düngebedarfs die im Boden vorhandenen Stickstoffgehalte nach $\$ 4$ Absatz 4 DüV nicht immer mittels repräsentativer Bodenproben zu ermitteln? ${ }^{78}$ Warum gibt es beim konventionellen Landbau anders als beim ökologischen Landbau ${ }^{79}$ keine rechtliche Obergrenze für den Viehbesatz, obwohl die zu hohen Überschüsse an Wirtschaftsdünger in einigen deutschen Regionen seit Jahrzehnten bekannt sind? Wie lassen sich bei der Düngeregulierung die örtlichen Standortverhältnisse stärker berücksichtigen? Gewährleistet das deutsche Düngerecht im Zusammenspiel mit dem übrigen Agrar- und Umweltrecht, dass internationale, europäische und verfassungsrechtliche Vorgaben zum Schutz der natürlichen Lebensgrundlagen nicht aufgrund landwirtschaftlicher Nährstoffeinträge verfehlt werden?

\section{Hauptaufgaben staatlicher Düngeregulierung und geeignete Instrumente}

Ökologisch wie rechtlich sind zwei Hauptaufgaben zu lösen: die Reduzierung der Gesamtnährstoffeinträge in die Umwelt und der ausreichende Schutz lokaler Ổosysteme vor übermäßigen Nährstoffeinträgen entsprechend ihrem Erhaltungszustand und ihrer spezifischen Vulnerabilität. Gesamtmengenreduzierung und standortgerechte Düngung sind sowohl ökologisch als auch rechtlich gebotene Notwendigkeiten (siehe 1.2 und 1.3). Hinzu kommt die Sicherstellung, dass zur Düngung nur geeignete Düngemittel ohne Schadstoffe gehandelt und eingesetzt werden. ${ }^{80}$ Die praktischen Möglichkeiten zur Reduzierung der Düngemengen bzw. der Nährstoffüberschüsse in der Landwirtschaft sind hierbei vielfältig, wie insbesondere der ökologische Landbau und die Techniken der Präzisionslandwirtschaft sowie Veränderungen der Tierbesatzdichten zeigen. ${ }^{81}$ Staatlicherseits

71) UN, Transforming our world: the 2030 Agenda for Sustainable Development - Resolution adopted by the General Assembly on 25 September 2015.

72) Bundesregierung, Deutsche Nachhaltigkeitsstrategie - Neuauflage 2016, 2016; BMEL, 2019 (Fn. 42); Bundesregierung, Klimaschutzplan 2050 - Klimaschutzpolitische Grundsätze und Ziele der Bundesregierung, 2019; Bundesregierung, 2019 (Fn. 13); Bundesregierung, Nationale Strategie zur Biologischen Vielfalt, 2007.

73) Taube, 2018 (Fn. 38)

74) Vgl. auch die Analyse von Douhaire, 2019 (Fn. 25), S. 148-153.

75) Ausführlich zur problematischen Freistellung dieser Kleingewässer Möckel/Bathe, Kleingewässer und Wasserrahmenrichtlinie Ist die deutsche Handhabung korrekt?, DVB1. 2013, $220 \mathrm{ff}$.

76) Zur Relevanz und zum Regulierungspotenzial z.B. Castellano u. a., Sustainable intensification of agricultural drainage, Nature Sustainability 2019.

77) Vgl. auch Douhaire, 2019 (Fn. 25).

78) Taube, 2018 (Fn. 38)

79) Vgl. Art. 15 Abs. 2 i.V.m. Anhang IV der EU-Verordnung 889/2008/EG.

80) Zur Qualitätssicherung durch europäisches und nationales Düngemittelrecht und diesbezügliche Defizite ausführlich Douhaire, 2019 (Fn. 25), S. 96-127.

81) Vgl. Häußermann и. а., 2019 (Fn. 5), S. 114 ff.; Thünen-Institut, 2019 (Fn. 8); Hülsbergen, Stickstoffeffizienz durch digitales Nährstoffmanagement und Precision Farming, 2019, https://www. acatech.de/wp-content/uploads/2019/05/H\%C3\%BClsbergen_ Stickstoffeffizienz_N\%C3\%A4hrstoffmanagement_PrecisionFarming.pdf; Finger u.a., Precision Farming at the Nexus of Agricultural Production and the Environment, Annual Review of Resource Economics 2019, https://doi.org/10.1146/annurev-resource-100518-093929; Bowles u.a. Nature Sustainability 2018, $399 \mathrm{ff}$.; Mueller u. a., Declining spatial efficiency of global cropland nitrogen allocation, Global Biogeochemical Cycles 2017; UBA, Umweltbelastende Stoffeinträge aus der Landwirtschaft: Möglichkeiten und Maßnahmen zu ihrer Minderung in der konventionellen Landwirtschaft und im ökologischen Landbau, 2015. 


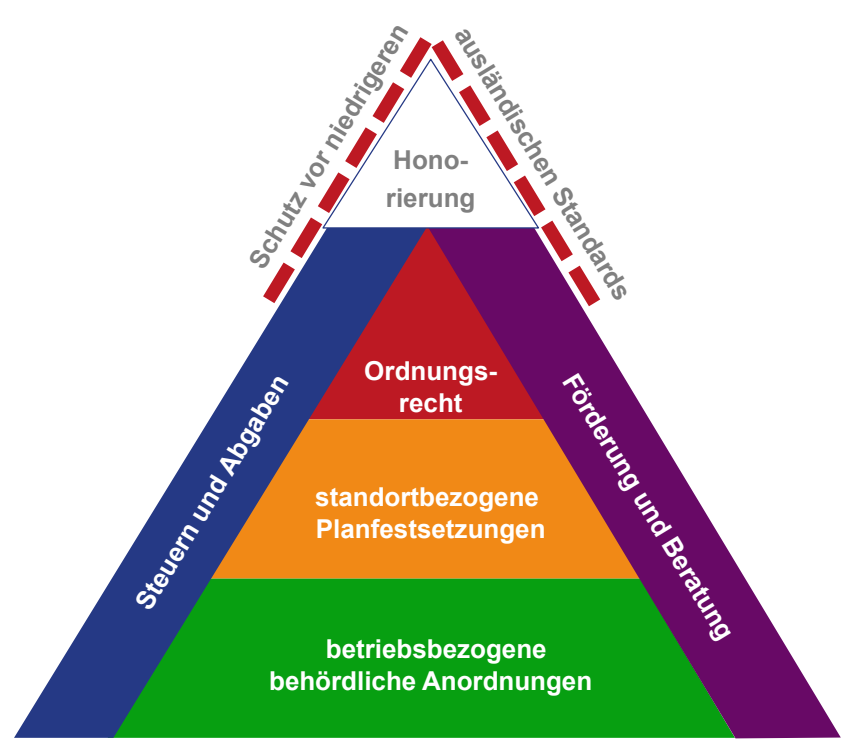

Abb. 2 Optimaler Instrumentenverbund zur staatlichen Lenkung der landwirtschaftlichen Bodennutzung Quelle: Eigene Darstellung

erfordert die Umsetzung der beiden Aufgaben einen breit aufgestellten Instrumentenmix (Abbildung 2). ${ }^{82}$ Ordnungs- und planungsrechtliche Instrumente sind dabei besonders gut geeignet, um flächendeckend allgemeinverbindliche Mindestanforderungen nach dem Verursacherprinzip aufzustellen, während die derzeit v.a. verwendeten Direktzahlungen als Beihilfen mit ökologischen Bewirtschaftungsauflagen hingegen weniger effektiv und effizient sind. ${ }^{83}$ Die schon heute sehr komplexe Düngeverordnung kann instrumentell den beiden Hauptaufgaben allein nicht gerecht werden. Es bedarf insbesondere ergänzender Anreizinstrumente, einer flächendeckenden ökologischen Betriebsberatung und standortbezogenen Konkretisierungen des Düngerechts auf Ebene der Länder oder Kommunen. ${ }^{84}$

\subsection{Instrumente zur Reduzierung der Gesamtnährstoffeinträge in die Umwelt}

Seit 1990 sind die Stickstoffüberschüsse für ganz Deutschland um ein Drittel auf rund $100 \mathrm{~kg}$ pro Hektar und Jahr zurückgegangen (siehe Abbildung 1). ${ }^{85}$ Auch für Phosphor gibt es einen abnehmenden Trend. ${ }^{86}$ Den größten Effekt hatten hierbei die strukturellen und wirtschaftlichen Veränderungen in Ostdeutschland im Zuge der Wiedervereinigung. Seitdem sind die Überschüsse im mehrjährigen Durchschnitt nur noch geringfügig gesunken und noch deutlich vom $70 \mathrm{~kg}$ Ziel der Bundesregierung $^{87}$ entfernt.

Der Rückgang ab 1995 wurde nicht durch eine Reduzierung des Einsatzes von Mineraldünger und Futtermitteln (Stickstoffzufuhr), sondern durch eine Steigerung der Erträge (Stickstoffabfuhr) und damit der Stickstoffeffizienz erreicht. Letztere wird allgemein als die zielführendste Maßnahme zur Verminderung der Stickstoffüberschüsse angesehen. ${ }^{88}$ Allerdings sind die Hektarerträge bei Getreidearten und Grünschnitt seit 2014 rückläufig (siehe Abbildung 3). Ob dies ein langfristiger Trend ist und welche Ursachen (z. B. Klimawandel, Resistenzen gegenüber Pestiziden, Rückgang der Bestäuber, Ausweitung des Ökolandbaus) ihm zu Grunde liegen, ist derzeit offen. Strengere düngerechtliche Bewirtschaftungsvorgaben können die Trendumkehr aber nicht erklären, da das nationale Düngerecht erst 2017 verschärft wurde.

Bei stagnierenden oder gar sinkenden Erträgen lassen sich die Nährstoffüberschüsse nur reduzieren und die Effizienz weiter steigern, indem man wie beim ökologischen Landbau die Nährstoffzufuhr verringert. Instrumentell lässt sich eine Reduzierung der gesamten Nährstoffüberschüsse und Einträge mit strengen ordnungsrechtlichen Vorgaben zu Art und Höhe des Einsatzes von Düngemitteln, zu Umfang und Art der Tierhaltung, zu Fruchtfolgen und Zwischenfrüchten sowie mittels verbindlicher Obergrenzen für Bilanzüberschüsse erreichen. ${ }^{89}$ Diesen Weg ist der Bundesgesetzgeber mit dem Düngerecht gegangen, wobei hier noch Luft nach oben besteht (siehe 2.). ${ }^{90}$ Die Vorgaben der $\mathrm{EU}^{91}$ zum ökologischen Landbau zeigen, dass auch durch ein Verbot von mineralischem Stickstoffdünger sowie einer Begrenzung des Viehbesatzes die Stickstoffüberschüsse sinken und die Effizienz ansteigt. ${ }^{92}$

Gesamtmengen lassen sich ebenfalls mittels ökonomischer Instrumente wie Steuern, Abgaben oder Zertifikate reduzieren, wenn sie entsprechende finanzielle Anreize setzen. ${ }^{93}$ In Folge des veränderten Kosten-Nutzen-Verhältnisses kommt es in der Praxis zu Verhaltensänderungen. Anders als bei ordnungsrechtlichen $\mathrm{Ge}$ - und Verboten bleiben dabei jedoch die Entscheidungen freiwillig und die verteuerten Verhaltensweisen weiterhin legal. Wegen dieser Steuerungsunschärfe im Einzelfall sind ökonomische Anreizinstrumente im Bereich der konkreten Gefahrenabwehr kein Ersatz für ordnungsrechtliche Vorgaben. Im Bereich der Gesamtsteuerung von Verhaltensweisen und insbesondere Ressourcennutzungen sind sie allerdings effizientere Instrumente, da wegen der Veränderung des ökonomischen Kalküls und der bleibenden Legalität des Verhaltens anders als bei Ge- und Verboten die einzelnen Verhaltensweisen keiner Kontrolle bedürfen. Zugleich verbessern Steuern, Abgaben und Zertifikate die Kostengerechtigkeit, wenn mit ihnen externe Kosten (z. B. aufgrund Umweltverschmutzung oder Gesundheitsbelastungen) den Verursachern angelastet werden. Auch wenn diese Internalisierung regelmäßig unvollständig und in pauschalierter Form möglich ist, hilft sie gleichwohl Vorteile auf Kosten der Allgemeinheit zu minimieren.

Mit Steuern oder Abgaben lassen sich daher der Gesamteinsatz von Düngemitteln bzw. die Gesamtnährstoff-

82) Vgl. Kanter u. a., Nitrogen pollution policy beyond the farm, $\mathrm{Na}$ ture Food 2020; Heißenhuber u. a., Position der Kommission Landwirtschaft beim Umweltbundesamt (KLU) (10/2019): Landwirtschaft quo vadis? Agrar- und Ernährungssysteme der Zukunft - Vielfalt gewähren, Handlungsrahmen abstecken, 2019; UBA, 2015 (Fn. 81); SRU, 2015 (Fn. 24).

83) Möckel, Verhältnis ordnungs- und beihilferechtlicher Mindestanforderungen im Agrarumweltrecht, ZUR 2016, 655 ff.; Möckel u. a., 2014 (Fn. 60), S. $357 \mathrm{ff}$.

84) Vgl. Garske, 2020 (Fn. 60); Möckel u. a., 2014 (Fn. 59).

85) Vgl. auch BMEL/BMU 2020 (Fn. 5), S. 82f., Tabelle 32, wobei seit 2009 wieder ein leichter kontinuierlicher Anstieg des Flächenbilanzsaldos zu beobachten ist, a.a.O., S. 85.

86) Vgl. Umweltbundesamt, Indikator: Eutrophierung von Flüssen durch Phosphor, https://www.umweltbundesamt.de/indikatoreutrophierung-von-fluessen-durch-phosphor\#die-wichtigstenfakten 2020; Garske, 2020 (Fn. 60).

87) Bundesregierung, 2016 (Fn. 72).

88) BMEL, 2019 (Fn. 43); Heißenhuber u. a., 2019 (Fn. 82). Außerhalb von Europa ist die Stickstoffeffizienz in den letzten Jahrzehnten allerdings gesunken (Mueller u. a. Global Biogeochemical Cycles 2017, $245 \mathrm{ff}$.)

89) Vgl. Garske, 2020 (Fn. 60); Möckel, Verbesserte Anforderungen an die gute fachliche Praxis der Landwirtschaft, ZUR 2014, $15 \mathrm{ff}$.

90) Vgl. die umfassende Defizitanalyse von Douhaire, 2019 (Fn. 25).

91) Ökolandbau-Verordnung 834/2007/EG und Durchführungsverordnung 889/2008/EG.

92) Thünen-Institut, 2019 (Fn. 8); UBA, 2015 (Fn. 81).

93) Garske, 2020 (Fn. 60); Möckel u. a., Einführung einer Abgabe auf Pflanzenschutzmittel in Deutschland, 2015; Gawel u. a., Weiterentwicklung von Abwasserabgabe und Wasserentnahmeentgelten zu einer umfassenden Wassernutzungsabgabe, 2011; Möckel, Umweltabgaben zur Ökologisierung der Landwirtschaft, 2006. 


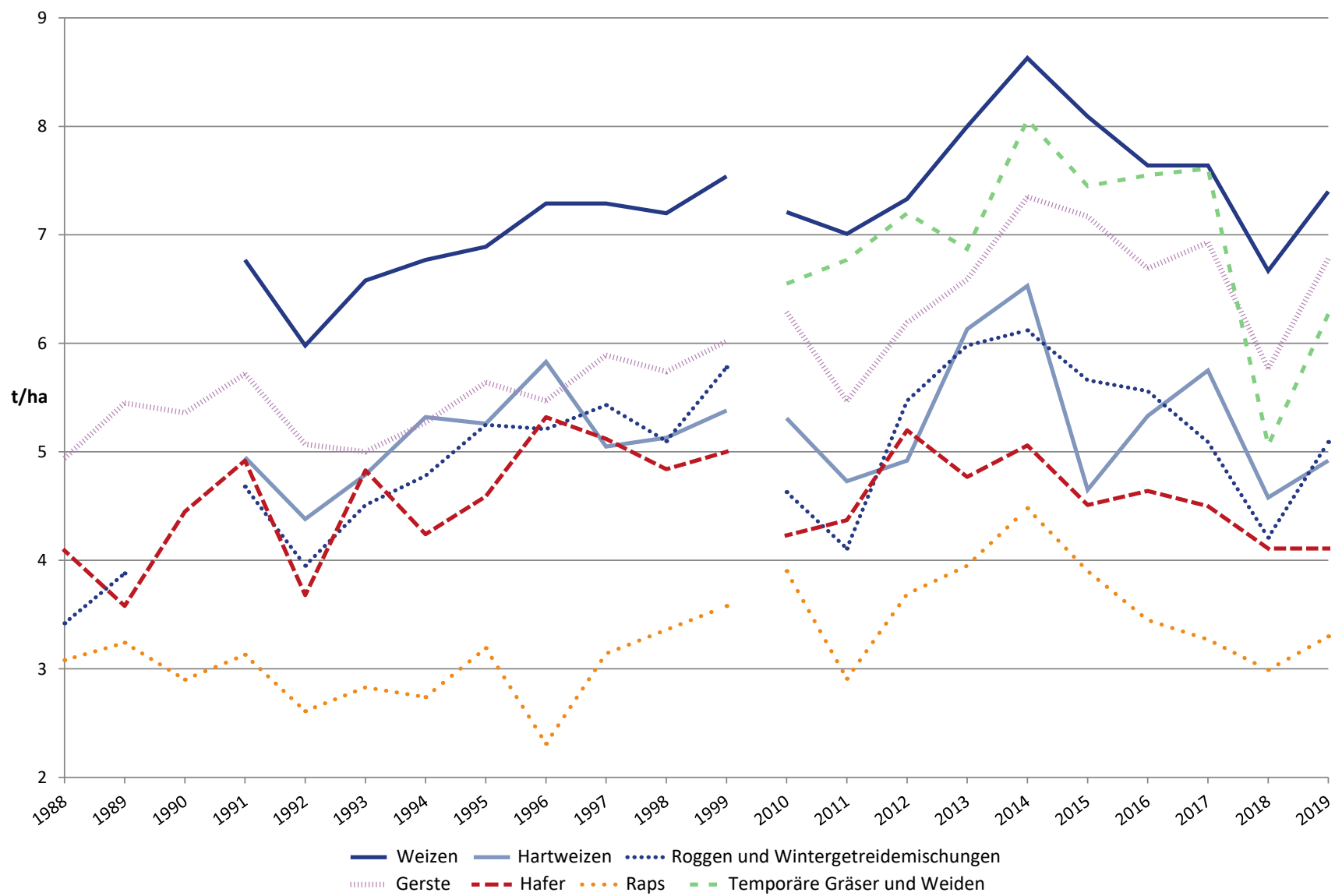

Abb. 3 Hektarerträge bei wichtigen Getreidearten und Grünschnitt in Deutschland von 1988 bis 2019

Quelle: Eigene Darstellung nach Daten von Eurostat, wobei zwischen 2000 bis 2009 für alle dargestellten Kulturen keine Daten verfügbar sind. Für Grünschnitt liegen vor 2010 keine Daten vor.

überschüsse effizient verringern und zugleich die gesellschaftlichen Umweltkosten der Nährstoffeinträge (z.B. für Klimaschäden, Trinkwasseraufbereitung und Naturschutzmaßnahmen) zumindest teilweise internalisieren. Nach Schätzungen betragen die externen Umweltkosten der deutschen Landwirtschaft mit 90 Mrd. EUR im Jahr das Vierfache der landwirtschaftlichen Bruttowertschöpfung, wobei ein erheblicher Teil der Kosten durch den Einsatz von Düngemitteln entsteht. ${ }^{94}$ Entsprechende Vorschläge werden auch zur Düngung schon länger in Deutschland diskutiert und gefordert. ${ }^{95}$ Es haben sich dabei zwei Wege herauskristallisiert. Zum einen lässt sich mit Verbrauchsteuern auf gehandelte Düngemittel und betriebsexterne Futtermittel (beides äußere Inputs zum lokalen Nährstoffkreislauf) der Einsatz bzw. der Anfall von Nährstoffen verteuern und damit reduzieren. Zum andern könnte man mittels einer Sonderabgabe auf die bilanziellen Nährstoffüberschüsse den Eintrag in die Umwelt bepreisen und diesbezügliche Minderungsanreize setzen. Die Unterschiede beider Ansätze sind in der Tabelle 1 dargestellt. Daneben besteht auch die Möglichkeit mit Lenkungsanreizen, das Ernährungsverhalten in der Bevölkerung zu verändern. So ließe sich mit einer steuerlichen Verteuerung tierischer Lebensmittel deren Nachfrage reduzieren und, solange Exportsteigerungen ${ }^{96}$ den inländischen Rückgang nicht ausgleichen, damit die Tierhaltung in Deutschland und die hierbei anfallenden Stickstoffemissionen und Wirtschaftsdüngermengen verringern. ${ }^{97}$

\subsection{Instrumente zum Schutz lokaler Ökosysteme}

Um Ökosysteme ausreichend vor übermäßigen Nährstoffeinträgen zu schützen, müssen sowohl die unterschiedlichen Wirkungen von Düngung und der eingesetzten
Düngemittel anhand der geologischen, topographischen, klimatischen und sonstigen ökologischen Standortverhältnisse als auch die verschiedenen Erhaltungszustände und spezifischen Vulnerabilitäten der im Ökosystem lebenden Tier- und Pflanzenarten berücksichtigt werden. Deutschlandweit einheitliche Vorgaben zur Art und Höhe der Düngung und zur Höhe von Bilanzüberschüssen genügen dem nicht. Vielmehr bedarf es einer rechtlichen Differenzierung, welche der ökologischen Diversität der Standorte soweit wie möglich gerecht wird.

Dies ist mit verschiedenen Instrumenten möglich und auch rechtlich zulässig. Nach der Rechtsprechung sowohl des Bundesverfassungsgerichts (BVerfG) als auch des Bundesverwaltungsgericht (BVerwG) sind aufgrund der Unvermehrbarkeit von Grund und Boden sowie seiner Situa-

94) Boston Consulting Group, Die Zukunft der deutschen Landwirtschaft nachhaltig sichern - Denkanstöße und Szenarien für ökologische, ökonomische und soziale Nachhaltigkeit, 2019.

95) Garske, 2020 (Fn. 60); Möckel, Rechtsgutachten zur Klärung von Rechtsfragen zur Erhebung einer Abgabe auf Stickstoffüberschuss und einer Abgabe auf stickstoffhaltigen Mineraldünger durch den Landesgesetzgeber, 2017; SRU, 2015 (Fn. 26), S. 30; Gawel u. a., 2011 (Fn. 92); SRU, Umweltgutachten 2004 - Umweltpolitische Handlungsfähigkeit sichern, 2004, Tz. $324 \mathrm{ff}$.; $M \ddot{o}-$ ckel, 2006 (Fn. 92).

96) UBA, 2018 (Fn. 4), S. $21 \mathrm{ff}$.

97) Vgl. Beermann u. a., Tierwohl fördern, Klima schützen - Wie eine Steuer auf Fleisch eine Wende in der Nutztierhaltung einleiten und Anreize für umweltschonenden Konsum liefern kann, Greenpeace 2020; Garske, 2020 (Fn. 60), S. 341 ff.; Wirz/Kasperczyk/Thomas, Ökologisierte Landwirtschaft in Deutschland 2050, Greenpeace 2016. 
Tab. 1 Vergleich von Abgaben auf externe Betriebsmittel und auf Nährstoffüberschüsse.

\begin{tabular}{|c|c|c|}
\hline Abgabe auf & externe Betriebsmittel & Nährstoffüberschüsse \\
\hline Charakter & $\begin{array}{l}\text { - Zielorientierung auf spezifische Störungen des loka- } \\
\text { len Nährstoffkreislaufs (Begin-of-Pipe Ansatz) } \\
\text { - Berücksichtigung von besonderen/herstellungsbe- } \\
\text { dingten Umweltauswirkungen möglich (z.B. CO } \text { CO }^{-} \\
\text {Bilanz von mineralischen Stickstoffdünger, Futter- }\end{array}$ & $\begin{array}{l}\text { - Zielorientierung auf die Überschüsse in die Umwelt (End-of- } \\
\text { Pipe Ansatz) } \\
\text { - keine Differenzierung nach Art und Herkunft des Nährstoffin- } \\
\text { puts möglich }\end{array}$ \\
\hline
\end{tabular}

Erhebungs- mineralische Düngemittel, zugekaufte Futtermittel gegenstände bzw. betriebsexterner Wirtschaftsdünger und Biogasabfälle (es sei denn Erzeugungs- und Ausbringungsflächen sind identisch), Klärschlämme

Rechtliche Verbrauchsteuer auf Verkaufs- bzw. Annahmepreise Form gemäß Art. 106 Abs. 1 Nr. 2 GG Alternativ: Verkehrsteuer nach Art. 106 Abs. 2 Nr. 3 GG

Verwaltungs- Gering, da Anknüpfung an Verkaufspreis wie bei anaufwand deren Verbrauchsteuern (z.B. MwSt, Energiesteuer, Alkopopsteuer).

Kontrollaufwand
Gering, da wie bei anderen Verbrauchsteuern Erhebung bei Händlern bzw. Herstellern erfolgt.
Überschuss nach Stoffstrombilanzierung ( $\$ 6$ StoffBilV) oder Flächenbilanzierung (ehemals $\$ 8$ DüV 2017)

(Finanzierungs)-Sonderabgabe ${ }^{98)}$ gemäß den Anforderungen des Bundesverfassungsgerichts: legitimer Sachzweck, Gruppenverantwortung der Abgabenschuldner, gruppennützige Verwendung des Aufkommens sowie keine überschießenden Einnahmen (vgl. BVerfG, B. v. 6.5.2014 - 2 BvR 1139/12, Rdnr. 115 ff.)

Hoch, da Überschussabgabe eine umfassende Ermittlung der Nährstoffströme im Betrieb bzw. je Fläche voraussetzt. Gering, wenn Bilanzierung schon ordnungsrechtlich vorgeschrieben ist (aktuell nur gemäß $\$ 1$ StoffBilV).

Hoch, da Kontrolle der Bilanzierung bei über 250000 Landwirten nötig.

Gering, wenn Bilanzierung schon ordnungsrechtlich vorgeschrieben ist, wobei aber Bilanzfälschungsinteresse mit Höhe der Abgabe ansteigt. tionsgebundenheit standortbezogene Vorgaben bei Grund und Boden gerechtfertigt ${ }^{99}$ oder wie das BVerwG formulierte: „Wenn die natürlichen oder landschaftsräumlichen Gegebenheiten eines Grundstücks im Interesse der Allgemeinheit erhaltenswert sind und des Schutzes bedürfen, so ergibt sich hieraus eine Art immanenter, d.h. dem Grundstück selbst anhaftender Beschränkung der Eigentümerbefugnisse, die durch natur- und landschaftsschutzrechtliche Regelungen lediglich nachgezeichnet wird." ${ }^{100}$

Auf die spezifischen Verhältnisse der jeweiligen Standorte kann im Rahmen von einzelbetrieblichen Beratungen ${ }^{101}$ und Förderungen eingegangen werden, wie sie gegenwärtig insbesondere im Rahmen der von der EU und dem Bund mitfinanzierten Agrarumweltprogramme der Länder erfolgen. Voraussetzung ist aber, dass der jeweilige landwirtschaftliche Betrieb ein entsprechendes Interesse hat. Aufgrund der Freiwilligkeit lässt sich mit diesen Maßnahmen - anders als mit ordnungsrechtlichen Ge- und Verboten - daher kein flächendeckender Schutz von Ökosystemen erreichen.

Differenzierte ordnungsrechtliche Vorgaben sind in verschieden Ausgestaltungsvarianten möglich. Zum einen lassen sich anhand von Standortmerkmalen (z.B. Bodentypen, Topografie, Niederschlagswerte, Biotoptypen und Arten sowie ihr Erhaltungszustand) Kategorien bilden, für welche dann spezifische Düngeanforderungen formuliert werden. Allerdings unterscheidet die DüV bisher lediglich zwischen stark humosen und weniger humosen Böden ( $\$ 4$ Abs. 1 Nr. 4 i.V.m. Tabelle 6 in Anlage 4) und differenziert sie die Gewässerabstände je nach Hangneigung ( $\$ 5$ Abs. 3). Dies ist durchaus ausbaufähig, wie die höheren standortspezifischeren Anforderungen bei den roten Gebieten gemäß $\ 13$ a DüV zeigen. Der Erhöhung der normativen Komplexität stünde dabei ein Gewinn an Handlungs- und Rechtssicherheit für den einzelnen Betrieb gegenüber.

Daneben existiert im Naturschutzrecht des Bundes und der Länder schon heute eine Auflistung von gesetzlich geschützten Biotopen, wobei allerdings auf der Rechtsfolgenseite bisher sehr allgemeine Schutzregeln stehen, die nur im Fall behördlicher Einzelanordnungen standortspezifisch konkretisiert werden (vgl. \$30 BNatSchG). In ergänzenden Gesetzesanhängen oder Verordnungen könnten für jeden Biotoptyp schon vom Gesetzgeber spezifische Schutzanforderungen normiert werden, um sowohl den Grundeigentümern und Bewirtschaftern als auch den Vollzugsbehörden wichtige Handreichungen zu geben. Die Bundesländer könnten hierbei vorangehen und nach $\iint 5$ Abs. 2, 30 Abs. 8, 44 Abs. 4 BNatSchG landesspezifische Vorgaben zur guten fachlichen Praxis, zum gesetzlichen Biotopschutz oder beim besonderen Artenschutz normieren. ${ }^{102}$

Zum anderen lassen sich ordnungsrechtliche Vorgaben an die Düngung auch räumlich spezifizieren. Ein erster dahingehender Schritt sind die roten Gebiete nach $\$ 13 \mathrm{a}$ DüV. Allerdings liegt ihr Fokus auf dem Gewässerschutz, weshalb unbefriedigende Erhaltungszustände und hohe Vulnerabilitäten bei terrestrischen Ökosystemen und Arten außen vor bleiben. Den Ländern bzw. den von ihnen ermächtigten Kommunen stehen aber verschiedene planungsrechtliche Möglichkeiten zur Verfügung, um auch bei terrestrischen Ökosystemen die Düngung standortspezifischer zu regulieren. ${ }^{103}$ Während Raumordnungspläne ( $\$ 7$ Raum-

98) Ausführlich Möckel, 2017 (Fn. 94).

99) BVerfG, Beschl. v. 22.5.2001 - 1 BvR 1512/97, 1677/97, BVerfGE 104, 1, 12; 12.1.1967 - 1 BvR 169/63, BVerfGE 21, 73, 82f.; BVerwG, 24.6.1993 - 7 C 26/92, BVerwGE 94, 1, 4 m.w. N.

100) BVerwG, Urt. v. 24.6.1993 - 7 C 26.92, BVerwGE 94, 1, 4 m. W. N.

101) Die Wirksamkeit von Beratung belegen Erfahrungen in Schweden und bei Pilotprojekten in Deutschland (vgl. SRU, 2015 (Fn. 26, Tz. 308)

102) Beim Dauergrünlandschutz ist dies u.a. in Baden-Württemberg, Mecklenburg-Vorpommern schon erfolgt. Ausführlich Möckel, Landesrechtliche Regelungsspielräume für ordnungsund planungsrechtliche Anforderungen an die landwirtschaftliche Bodennutzung, DÖV 2017, 192.

103) Eine ausführliche Darstellung der einzelnen Planungsinstrumente findet sich in Möckel u. a., 2014 (Fn. 60), S. 389-426. 
ordnungsgesetz), Landschaftspläne ( $\int S 10,11$ BNatSchG) sowie Maßnahmenprogramme und Bewirtschaftungspläne (\$S $82 \mathrm{f}$. WHG) mehr der verwaltungsinternen Koordinierung dienen, lassen sich bei folgenden planerischen Instrumenten außenverbindliche Anforderungen zur Bewirtschaftung und Düngung festsetzen: natur-, wasser- oder bodenschutzrechtliche Schutzgebiete oder -objekte nach \$S 20-29 BNatSchG, \$S $51 \mathrm{f}$. WHG und $\$ 21 \mathrm{Abs}$. 3 BBodSchG, Überschwemmungsgebiete gemäß $\$ 78$ WHG sowie in NRW und Berlin örtliche Landschaftspläne i.S.v. \11 BNatSchG.

Hinsichtlich der Natura 2000 Schutzgebiete (\$S 31-36 BNatSchG) ist spätestens mit dem EuGH Urteil ${ }^{104}$ vom November 2018 offensichtlich geworden, dass aus Art. 6 FFH-Richtlinie auch Anforderungen an die landwirtschaftliche Bodennutzung (einschließlich Düngung und Beweidung) resultieren, welche die Länder in ihren Schutzgebietsvorschriften und Managementplänen verankern müssen. Solange dies nicht erfolgt ist, sind Düngemaßnahmen und Weidehaltungen in oder in der Nähe von Natura 2000 Gebieten gemäß $\$ 34$ Abs. 6 BNatSchG vorher anzuzeigen, damit die zuständige Behörde eine FFH-Vorprüfung und gegebenenfalls Hauptprüfung durchführen kann.

Für die rein nationalen Schutzgebiete haben die zuständigen Behörden einen weiten Ermessensspielraum, ob und mit welchen Schutzregelungen sie ein Gebiet unter Schutz stellen. Bei Wasserschutzgebieten sieht $₫ 51$ Abs. 1 Nr. 3 WHG ausdrücklich auch eine Unterschutzstellung vor, um den Eintrag von Bodenbestandteilen, Dünge- oder Pflanzenschutzmitteln in Gewässer zu vermeiden. Allerdings wird bei einer Vielzahl der wasser- und naturschutzrechtlichen Schutzgebiete die ordnungsgemäße Landwirtschaft nach guter fachlicher Praxis in den Ländern freigestellt. ${ }^{105}$ Bei Wasserschutz- und Überschwemmungsgebieten dürften die finanziellen Ausgleichspflichten der Länder für darüber hinausgehende Beschränkungen der landwirtschaftlichen Bodennutzung nach $\$ \int 52$ Abs. 5, 78 Abs. 5 WHG ein wesentlicher Grund hierfür sein. Da ein solcher Ausgleich für durch öffentliche Zwecke gerechtfertigte, zumutbare Beschränkung der Berufs- und Eigentümerfreiheiten verfassungsrechtlich nicht geboten ist, empfiehlt sich die Aufgabe dieser gleichheitsrechtlich problematischen Privilegierung.

Schutzgebiete dürfen allerdings nur für Flächen ausgewiesen werden, die schutzwürdig und schutzbedürftig sind. Für andere Flächen sind verbindliche planungsrechtliche Vorgaben derzeit nur möglich, wenn die Länder für kommunale Landschaftspläne eine Außenverbindlichkeit gemäß $\$ 11$ Abs. 1 BNatSchG vorsehen. Ansonsten bleibt die kommunale Planungshoheit derzeit nach dem Baugesetzbuch auf die Bauleitplanung beschränkt, da in Bebauungsplänen nur Art und Maß der baulichen Nutzung festgesetzt werden können. Verfassungsrechtliche, demokratische und ökologische Gründe sprechen dafür, derartige kommunale Steuerungsmöglichkeiten auch für alle nichtbaulichen Flächen gesetzlich vorzusehen, da insbesondere die land- und forstwirtschaftlichen Flächen regelmäßig den größten Teil des Gemeindegebietes ausmachen und Lebensumfeld der Gemeindebürger sind. ${ }^{106}$ Der Bund könnte diese Möglichkeit mit einer Erweiterung der Bauleitplanung zu einer Bodennutzungsplanung oder einer Außenverbindlichkeit für örtliche Landschaftspläne relativ einfach einführen. Es wären dann in ganz Deutschland standortbezogene Konkretisierungen der guten landwirtschaftlichen Praxis möglich.

Zur Durchsetzung und betriebsbezogenen Feinjustierung sind die ordnungs- und planungsrechtlichen Anforderungen mit entsprechenden behördlichen Anordnungsbefugnissen und gegebenenfalls auch Anzeigepflichten und Genehmigungsvorbehalten auszustatten. ${ }^{107}$ Ergänzend empfiehlt es sich zur Minderung von unvermeidbaren Be- einträchtigungen der Leistungs- und Funktionsfähigkeit des Naturhaushaltes durch landwirtschaftliche Bodennutzungen einschließlich unvermeidbarer Nährstoffüberschüsse eine pauschalisierte Eingriffskompensation (z.B. in Form eines Mindestanteils ökologischer Vorrangflächen oder produktionsintegrierter Maßnahmen) einzuführen, um zu vermeiden, dass aufgrund der allgemein wiederlegten Regelvermutung umfangreiche Einzelfallprüfungen durchzuführen sind. ${ }^{108}$

\section{Fazit}

Die neuesten Veränderungen in der DüV verbessern das Düngerecht an einigen Stellen und verschärfen insbesondere die düngerechtlichen Anforderungen in den roten Gebieten. Die ersatzlose Streichung der Flächenbilanzobergrenzen könnte aber bei vielen landwirtschaftlichen Flächen sogar zu höheren Nährstoffeinträgen in die Umwelt führen, da die Stoffstrombilanzverordnung nicht nachgebessert wurde. Ob daher die Ziele der Nitrat-Richtlinie tatsächlich auch insgesamt erreicht werden, bleibt abzuwarten und hängt auch vom Vollzug der Länder ab.

Fest steht schon jetzt: die Novelle und die Diskussion greifen mit ihrer Fokussierung auf die Nitrat-Richtlinie und den Gewässerschutz insgesamt zu kurz, da sie die weitreichenden ökologischen Auswirkungen von Nährstoffüberschüssen und diesbezügliche Schutzverpflichtungen Deutschlands nicht umfassend in den Blick nehmen. Hierzu gehören neben den Klimawirkungen insbesondere die zu hohen Nährstoffeinträge in terrestrische Ökosysteme, welche die Artenzusammensetzungen verändern und die Erreichung internationaler, europäischer sowie nationaler Naturschutzziele gefährden.

Eine dauerhaft umweltgerechte Düngung ist eine Grundbedingung für eine insgesamt nachhaltige Landwirtschaft. In Anbetracht der langfristigen ökologischen Folgen einer übermäßigen Düngung ist eine wesentlich größere Reduktion der Nährstoffüberschüsse als bisher notwendig. Dies gilt sowohl hinsichtlich der Gesamtmengen als auch hinsichtlich der spezifischen Einträge in lokale Ökosysteme.

Open Access. Dieser Artikel wird unter der Creative Commons Namensnennung 4.0 International Lizenz veröffentlicht, welche die Nutzung, Vervielfältigung, Bearbeitung, Verbreitung und Wiedergabe in jeglichem Medium und Format erlaubt, sofern Sie den/die ursprünglichen Autor(en) und die Quelle ordnungsgemäß nennen, einen Link zur Creative Commons Lizenz beifügen und angeben, ob Änderungen vorgenommen wurden.

Die in diesem Artikel enthaltenen Bilder und sonstiges Drittmaterial unterliegen ebenfalls der genannten Creative Commons Lizenz, sofern sich aus der Abbildungslegende nichts anderes ergibt. Sofern das betreffende Material nicht unter der genannten Creative Commons Lizenz steht und die betreffende Handlung nicht nach gesetzlichen Vorschriften erlaubt ist, ist für die oben aufgeführten Weiterverwendungen des Materials die Einwilligung des jeweiligen Rechteinhabers einzuholen.

Weitere Details zur Lizenz entnehmen Sie bitte der Lizenzinformation auf http://creativecommons.org/licenses/by/4.0/deed.de.

Open Access funding enabled and organized by Projekt DEAL.

104) EuGH, Urt. v. 7.11.2018 - C-293/17 und C-294/17. Ausführlich zu den Konsequenzen für die Landwirtschaft Möckel NuR 2019, $152 \mathrm{ff}$.

105) Auswertung der Datenbank JURIS.

106) Ausführlich Möckel, Erfordernis einer umfassenden außenverbindlichen Bodennutzungsplanung auch für nichtbauliche Bodennutzungen, DÖV 2013, $424 \mathrm{ff}$.

107) Ausführlich Möckel in: Möckel u. a., 2014 (Fn. 60), S. 427-462.

108) Möckel NuR 2018, 742 ff.; Möckel NuR 2012, 225 ff. 